\title{
The possible statistical relation of Pc1 pulsations to Earthquake occurrence at low latitudes
}

\author{
J. Bortnik ${ }^{1}$, J. W. Cutler ${ }^{2}$, C. Dunson ${ }^{3}$, and T. E. Bleier ${ }^{3}$ \\ ${ }^{1}$ Dept. of Atmospheric and Oceanic Sciences, Room 7115 Math Sciences Bldg., UCLA, Los Angeles, CA 90095-1565, USA \\ ${ }^{2}$ Department of Aerospace Engineering, 1320 Beal Ave., 3013 FXB Building, Ann Arbor, MI 48109-2140, USA \\ ${ }^{3}$ QuakeFinder LLC., 250 Cambridge Ave., Suite 204, Palo Alto, CA. 94305, USA
}

Received: 4 April 2008 - Revised: 4 August 2008 - Accepted: 19 August 2008 - Published: 22 September 2008

\begin{abstract}
We examine the association between earthquakes and Pc1 pulsations observed at a low-latitude station in Parkfield, California. The period under examination is $\sim 7.5$ years in total, from February 1999 to July 2006, and we use an automatic identification algorithm to extract information on Pc1 pulsations from the magnetometer data. These pulsations are then statistically correlated to earthquakes from the USGS NEIC catalog within a radius of $200 \mathrm{~km}$ around the magnetometer, and $M>3.0$. Results indicate that there is an enhanced occurrence probability of Pc1 pulsations $\sim 5-15$ days in advance of the earthquakes, during the daytime. We quantify the statistical significance and show that such an enhancement is unlikely to have occurred due to chance alone. We then examine the effect of declustering our earthquake catalog, and show that even though significance decreases, there is still a statistically significant daytime enhancement prior to the earthquakes. Finally, we select only daytime Pc1 pulsations as the fiducial time of our analysis, and show that earthquakes are $\sim 3-5$ times more likely to occur in the week following these pulsations, than normal. Comparing these results to other events, it is preliminarily shown that the normal earthquake probability is unaffected by geomagnetic activity, or a random event sequence.
\end{abstract}

Keywords. Ionosphere (Mid-latitude ionosphere) - Magnetospheric physics (Plasma waves and instabilities) - Radio science (Ionospheric propagation)

\section{Introduction}

Non-seismic precursors to earthquakes have been reported in the literature as early as the 1950s (Kalashnikov, 1954), and take on a variety of forms. Viewed as a whole, such

Correspondence to: J. Bortnik

(jbortnik@gmail.com) reported precursors can occupy almost any frequency range spanning from quasi-DC to visible light (e.g. Derr, 1973; Johnston, 1989; Parrot and Johnston, 1989; Park et al., 1993), occur over a range of timescales preceding the main shock spanning from minutes (Parrot and Lefeuvre, 1985) to years (Zhao and Qian, 1994), and exhibit a range of morphologies which includes signal enhancement, attenuation, modulation, and spikes, in a bewildering variety of signals including electric, magnetic, and electromagnetic signals, resistivity changes, anomalous ionospheric variations, radon gas release, and others (e.g. Hayakawa et al., 2006, for a special issue dealing with recent progress in this area).

Perhaps it is not surprising then, that there have also been studies that report no correlation, or only occasional correlation between earthquakes and some of the phenomena previously reported as precursors (Hendersen et al., 1993; Rodger et al., 1996, 1999; Clilverd et al., 1999). This lack of correlation could possibly be due to the fact that any potential precursors are strongly dependent on the local geology of the hypocenter (and hence appear differently in different regions, depths, and even times), they could be swamped by the natural background signal environment, may not occur in a repeatable way, or may not occur at all. To complicate matters further, even though many theoretical models to explain precursory phenomena have been proposed over the years (e.g. Brace et al., 1965; Nagata, 1970a, b; Hanks, 1974; Ishido and Mizutani, 1981; Dogloglou-Revelioti and Varotsos, 1986; Morgan et al., 1989; Park et al., 1993; Molchanov et al., 2001; Pulinets and Boyarchuk, 2004; Freund, 2007) the mechanisms responsible for earthquake precursors are still understood rather poorly, making it difficult for experimentalists to conduct targeted observational campaigns.

Studies conducted to date have necessarily taken one of two approaches: (i) placing an instrument in a fixed location on the Earth's surface, in the hopes that an earthquake will occur sufficiently close-by during the period of

Published by Copernicus Publications on behalf of the European Geosciences Union. 


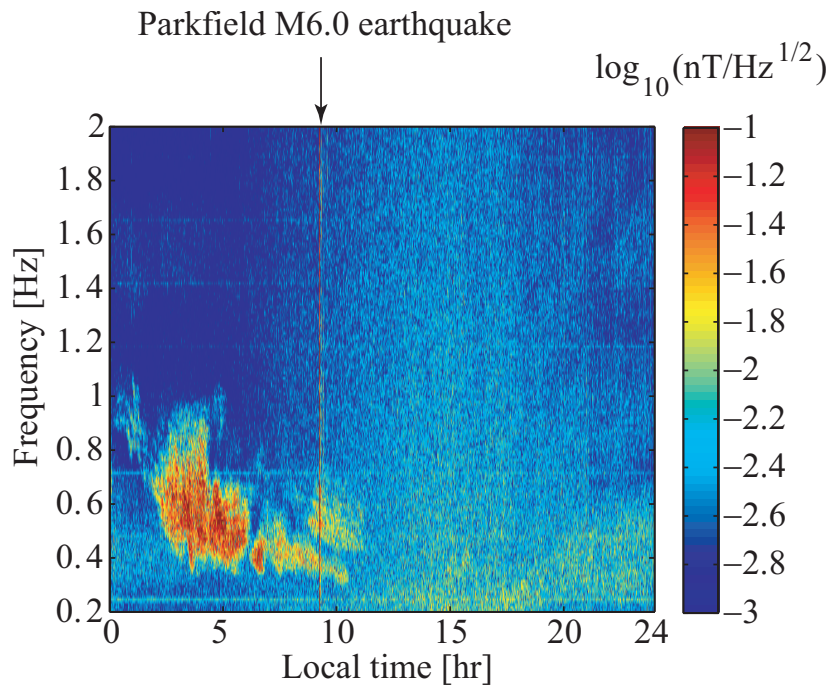

Fig. 1. Example of a Pc1 pulsation observed at the PKD search-coil magnetometer (see text) shortly before the M6.0 Parkfield earthquake on 28 September 2004, 10:15:24 (PDT). The intense Pc1 pulsation is visible between 03:00-11:00 LT, and $f=0.4-0.8 \mathrm{~Hz}$. We note that local time is taken to be Pacific Standard Time (PST, i.e. UT-8) with no provision for daylight time adjustment.

observation (e.g. Fraser-Smith et al., 1990; Bernardi et al., 1991; Hayakawa and Molchanov, 2004; Hayakawa et al., 2007), and (ii) placing an instrument aboard an orbiting satellite, and thus observing the effects of numerous earthquakes (e.g. Larkina et al., 1983; Parrot and Lefeuvre, 1985; Parrot, 2006). The advantage of (i) over (ii), is that a specific region can be observed continuously over a long time-period, and hence the natural background signal environment can be carefully assessed, and anomalous activity (potentially associated with earthquakes) more readily recognized. Another advantage is that the earthquakes in a given region would, presumably, all behave in a roughly similar manner (in terms of precursors), and hence some sort of repeatable precursory behavior might be identified. On the other hand, given the capricious nature of earthquakes, there might not ever be a large earthquake sufficiently close to the instrument to be in any way observable, or there may only be very few events if the instrument is operated for a long time. This naturally forces ground-based observationalists to conduct more eventbased studies, which, while certainly suggestive, lack the assurance of statistically significant repeatability. On the other hand, the advantage of (ii) is that the entire globe is essentially sampled for earthquakes on a daily basis, and hence many events could be observed in a relatively short period of time. The disadvantage of (ii) is that the satellite spends only a short period of time in any given location, so the earthquakes are sampled only briefly from a variety of epicentral locations, possibly introducing large heterogeneity in precursory morphologies, and also earthquakes that might not exhibit any precursory behavior at all.
In an attempt to use the advantages of both approaches (long-term local characterization with a large number of events), in the present paper, we study a $\sim 7.5$ year record from a ground-based, search-coil magnetometer located in Parkfield, California, for possible association with earthquakes. We focus our study on a particular signal type, Pc1 pulsations (Sect. 2), a common signal recorded at Parkfield. We use an identification algorithm to automatically extract Pc1 pulsations (Bortnik et al., 2007) from our data (Sect. 3.1), which are then treated as point processes, and using the statistical machinery of point processes, are related to earthquake occurrence within a certain radius of the magnetometer (Sect. 4). We then attempt to quantify the significance of our correlation (Sect. 5), and examine the effects upon this significance of declustering our earthquake cata$\log$ (Sect. 6). We invert our superposed epoch analysis to calculate earthquake probability relative to Pc1 occurrence (Sect. 8), and summarize our conclusions (Sect. 9).

\section{Background}

An example of the signal type under study, a Pc1 pulsation, is shown in Fig. 1. It consists of a continuous magnetic pulsation in the frequency range $f=0.2-5 \mathrm{~Hz}$ (defined as the Pc1 frequency band), usually lasting in the range of minutes to hours (Kangas et al., 1998). This pulsation began several hours prior to the M6.0 Parkfield earthquake that occurred on 28 September 2004 at 10:15:24 local time (PDT), shown in the figure as a vertical line at 09:15:24 (PST).

The classical view of the origin and propagation of $\mathrm{Pc} 1$ pulsations (e.g. Jacobs, 1970) is illustrated in Fig. 2. Electromagnetic ion cyclotron (EMIC) waves are generated in the equatorial region of the magnetosphere at $L \sim 4-8$ (Anderson et al., 1992) due to freshly-injected, anisotropic distributions of ring-current ions (Cornwall, 1965), that are unstable to the growth of Left-hand (L)-mode waves, as shown in Fig. 2a-1 (although other sources of instability have also been identified (Olson and Lee, 1983; Kangas et al., 1986; Engebretson et al., 2002)). The EMIC waves propagate roughly along the magnetic field line in the L-mode (Fig. 2a-2), possibly undergoing mode-conversion and mode-coupling at higher latitudes (Johnson and Cheng, 1999) and enter the highlatitude ionosphere (Fig. 2a-3), in what is sometimes referred to as the secondary source (e.g. Webster and Fraser, 1984). The EMIC waves then couple to the R-mode and propagate horizontally and isotropically (Fig. 2b-4) in the ionospheric waveguide (e.g. Manchester, 1966, 1968; Fraser, 1968; Jacobs, 1970 , p. 115), which is centered on the $\mathrm{F} 2$ region electron density maximum at $\sim 350 \mathrm{~km}$ (Manchester, 1966). The wave power progressively attenuates and leaks to the ground through the lossy bottomside ionosphere as it propagates from high to low latitudes, the attenuation being most severe during the daytime (Althouse and Davis, 1978), resulting in a nighttime occurrence maximum at low latitudes (e.g. Jacobs, 
1970, p. 28), and concomitant minimum during the day. At low latitudes, Pc1 occurrence typically maximizes in the 2-7 days following the main phase of geomagnetic storms (Wentworth, 1964; Heackock and Kivinen, 1972). The number, frequency, and diurnal distribution of Pc1 pulsations also depends on a variety of factors including solar cycle phase, storm intensity, observation latitude, time after main phase, and pulsation type (structured/unstructured) (e.g. Kerttula et al., 2001a, b).

In this paper, we adopt as a hypothesis, the conceptual model that earthquake preparation processes alter the local ionosphere overhead, which in turn dramatically affects the transmission coefficient of the bottomside ionosphere (Field and Greifinger, 1965, 1966). This alteration leads to a change in the number and nature of $\mathrm{Pc} 1$ pulsations that leak through the ionosphere and are observed on the ground. The precursory effect of earthquakes on the local ionosphere overhead has been reported by a number of authors (e.g. Pulinets et al., 2003; Pulinets and Boyarchuk, 2004, for reviews), and since Pc1 transmission is very sensitive to bottomside characteristic, it can serve as a sensitive proxy to ionospheric modifications. Of course, this model is in no way definitive and any potential modification of Pc1 pulsations could occur in other ways, including being directly generated by strong electric currents near the hypocenter itself (Morgan et al., 1976; Ishido and Mizutani, 1981; Dogloglou-Revelioti and Varotsos, 1986; Freund, 2007).

\section{Data description and Pc1 identification}

\subsection{Pc1 identification}

To identify Pc1 pulsations, we use triaxial search-coil magnetometer data recorded at Parkfield, California (designated PKD), whose coordinates are: Geographic: $\left(35.945^{\circ}\right.$, $\left.-120.542^{\circ}\right)$, CGM: $\left(41.61^{\circ},-56.8^{\circ}\right)$, dip: $60.2^{\circ}$, declination: $14.7^{\circ}$, L-value: 1.77. We use data from the $\sim 7.5$ year period, February 1999-July 2006. The Pc1 pulsations are automatically identified and characterized using a recently developed algorithm, described in detail by Bortnik et al. (2007). This algorithm consists of three broad steps:

1. Spectral peaks in a typical daily, dynamic spectrogram are identified, which exceed the daily background median spectrum significantly (by a factor of $\sim 10$ in our case). Three key frequencies are recorded to describe each spectral peak: the bottom $\left(f_{\mathrm{bot}}^{\mathrm{pk}}\right)$, top $\left(f_{\mathrm{top}}^{\mathrm{pk}}\right)$, and the frequency containing maximum wave power $\left(f_{\max }^{\mathrm{pk}}\right)$.

2. The spectral peaks in consecutive time-segments in the dynamic spectrogram are grouped together to form individual events. Spectral peaks need to satisfy a "spectral overlap" criterion, and be of a certain minimum duration (10 min in our case) to be counted as an event. Typical Pc1 events have durations from fractions of an

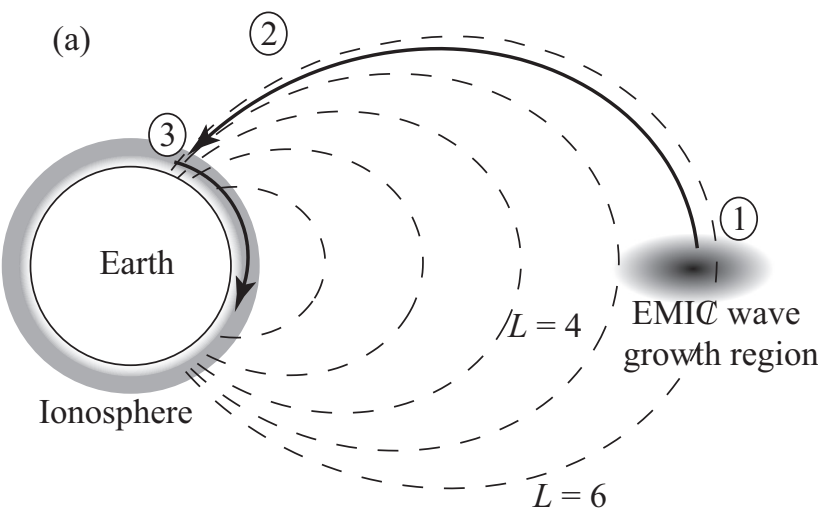

(b)

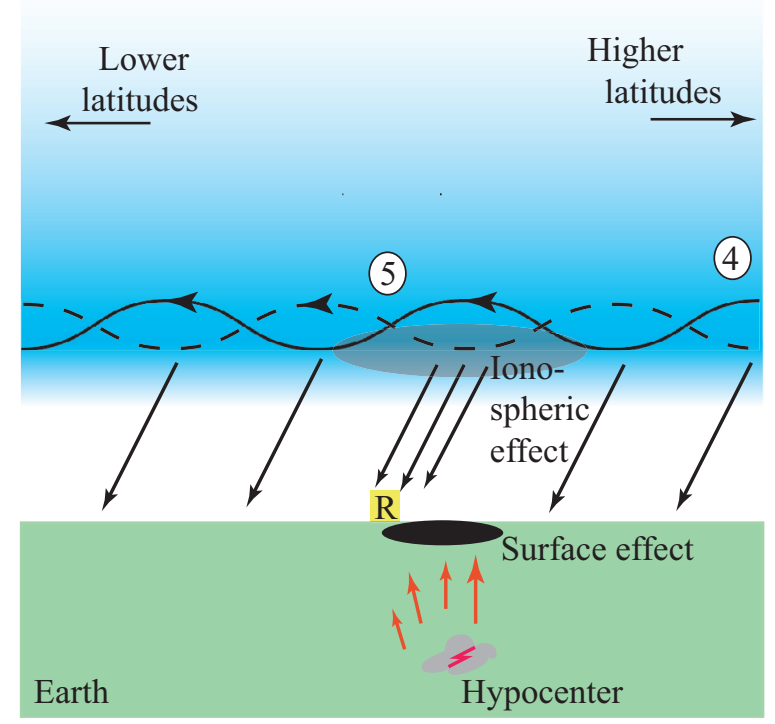

Fig. 2. A possible mechanism of Pc1 modulation by Earthquakes. (a) The electromagnetic ion cyclotron (EMIC) wave is generated near the equatorial region in the magnetosphere (1), propagates roughly along the magnetic field line (2), enters the ionosphere at high latitudes (3) and propagates within the dense F-region of the ionosphere to low-latitudes. (b) During its meridional propagation to low-latitudes (4), seismically active regions might affect the ionosphere in such a way as to change the bottomside transmission coefficient (5), resulting in anomalous Pc1 activity recorded at the receiver station $\mathrm{R}$.

hour, to several hours, and spectral peaks shorter than $10 \mathrm{~min}$ are treated as be spurious noise in our analysis and discarded.

3. The full set of polarization parameters (e.g. polarization ratio $R_{\text {pol }}$, ellipticity $\tan (\beta)$, sense of rotation $(\operatorname{sign}(\beta))$, major axis orientation $\left(\tan \left(\theta_{\mathrm{ax}}\right)\right)$, and wave normal angles $\left.\left(\theta_{\mathrm{k}}, \phi_{\mathrm{k}}\right)\right)$ are extracted for each of the identified wave events, and recorded as a function of time. 

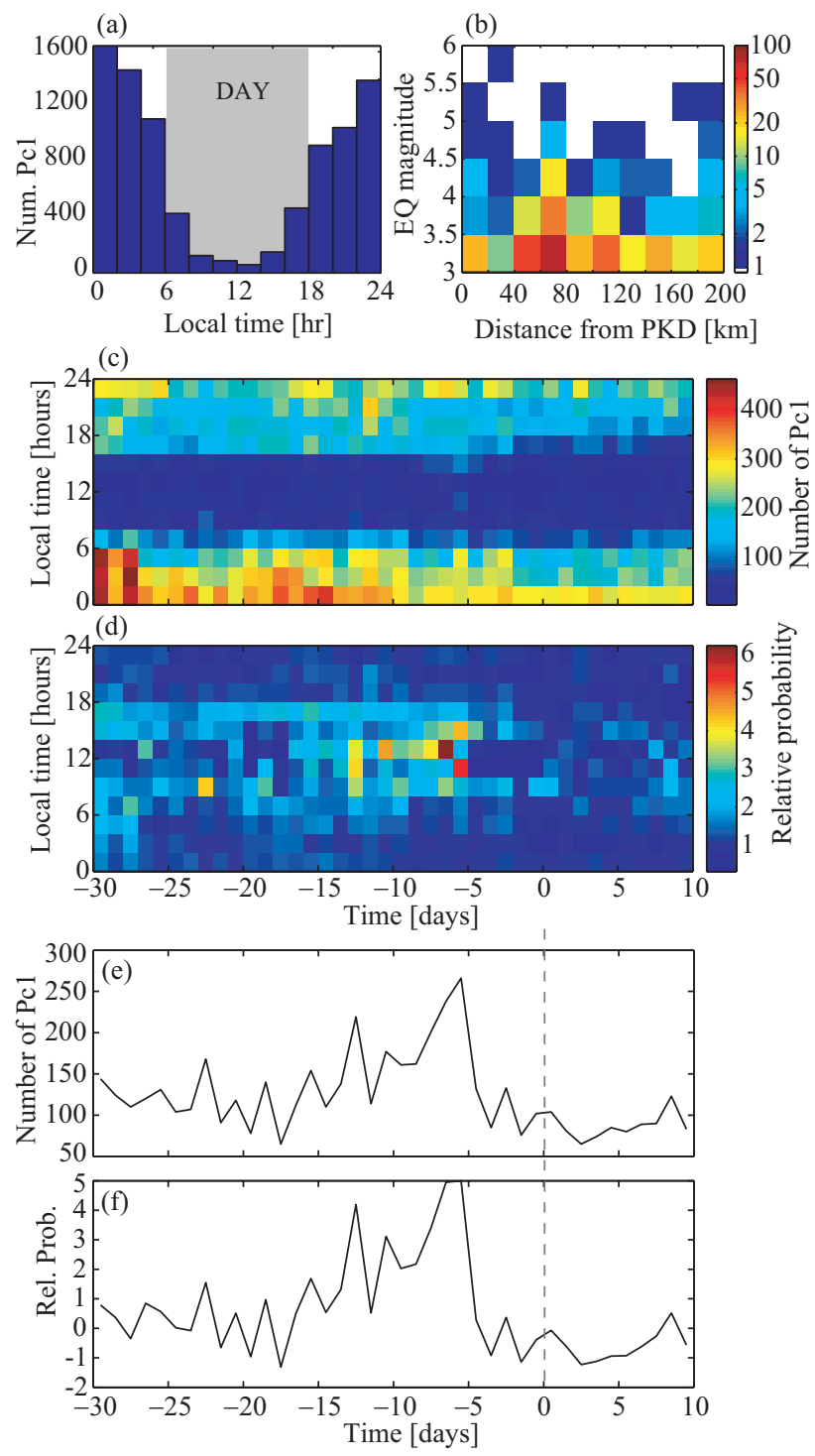

Fig. 3. (a) Distribution of 8,193 Pc1 pulsations as a function of local time. (b) Distribution of 434 earthquakes during the period under consideration, as a function of magnitude, and distance from our search-coil. (c) Superposed epoch analysis of Pc1 events observed within -30 to +10 days of our 434 earthquakes, as a function of $\Delta T$ and LT. (d) Relative probability of observing Pc1 pulsations in the same format as (c). (e) Number of Pc1 pulsations observed on the day side, 08:00-16:00 LT, corresponding to panel (c). (f) Same as (e), but corresponding to panel (d).

Although our Pc1 identification algorithm was developed for the full triaxial data set, and we have all three components available for the period under study, in the present work we have chosen to use only the two horizontal channels for Pc1 identification/characterization. This was done because we do not use polarization or orientation information in the present study (which would necessitate the vertical channel), and re- sults in more robust Pc1 identification since the vertical coil is often more susceptible to local noises and contamination from underground reflections.

A total of 8,913 individual Pc1 events were identified in our $\sim 7.5$ year period, having a broad local-time (LT) occurrence minimum at $\sim 08: 00-15: 00$, and maximum at $\sim 23: 00$ 04:00. This is consistent with previous studies (Tepley, 1965) reported earlier (Bortnik et al., 2007) and shown in Fig. 3a. The mean duration of Pc1 events was $\sim 20 \mathrm{~min}$, and $f_{\mathrm{max}}^{\mathrm{pk}}$ ranged from $0.12-4.7 \mathrm{~Hz}$, with a median and mean value of 0.44 and $0.8 \mathrm{~Hz}$, respectively.

In this paper we present information only on Pc1 occurrence, neglecting duration, frequency, and polarization information. The latter quantities were examined for any statistical deviation from the norm, and none was found.

\subsection{Earthquake catalog}

In order to correlate Pc1 pulsations to nearby earthquakes, we used the United States' Geological Survey (USGS) National Earthquake Information Center (NEIC) earthquake catalog (more information at http://neic.usgs.gov) to retrieve earthquake events occurring over the same period as our magnetometer data, and within a large radius $(\sim 3000 \mathrm{~km})$ surrounding our magnetometer. In subsequent sections, only earthquakes located within a radius of $200 \mathrm{~km}$ were used, but the inclusion of the larger initial radius was made in order to facilitate declustering, discussed in Sect. 6.

\section{Superposed epoch analysis}

In order to examine the association between Pc1 occurrence and earthquakes, we perform a superposed epoch analysis spanning -30 days to +10 days about the earthquake time, comparable to a second-order moment in point-process statistics (e.g. Hsu and McPherron, 2002). For this purpose, we select earthquakes whose epicenters were within $200 \mathrm{~km}$ of the PKD search-coil, magnitudes larger than 3.0, and depth smaller than $50 \mathrm{~km}$. This results in a list of 434 earthquakes, spanning February 1999-May 2006, with a minimum and maximum magnitudes of 3.1 and 6 , median 3.5, minimum and maximum distances from PKD of $2.2 \mathrm{~km}$ and $200 \mathrm{~km}$, median $72.6 \mathrm{~km}$. The distribution of earthquakes is shown in Fig. $3 \mathrm{~b}$ as a function of distance and magnitude, where it is evident that earthquake occurrence diminishes rapidly with increasing magnitude.

Using the mean local time (LT), and mean universal time (UT) of each Pc1 pulsation, we calculate the time-difference $(\Delta \mathrm{T})$ of each Pc1 pulsation relative to each of the earthquake times in our selected list, and bin the pulsations as shown in Fig. 3c into $2 \mathrm{~h}$ LT bins (i.e. 12 total LT bins, ordinate of Fig. 3c), and $24 \mathrm{~h}$ relative-time bins (i.e. 1 bin per day, abscissa of Fig. 3c). The number of Pc1 pulsations in each $\triangle \mathrm{T}$-LT bin is shown in color, and indicates that there is a 
pronounced tendency of Pc1 pulsations to be observed in the night and dawn hours, due primarily to the larger degree of ionospheric attenuation experienced by $\mathrm{Pc} 1$ pulsations as they propagate from high latitudes to lower latitudes on the day side (e.g. Althouse and Davis, 1978; Jacobs, 1970, p. 28). From Fig. $3 \mathrm{c}$ there also appears to be no clear relation between the earthquake time and Pc1 occurrence (although a slight decrease in events in the -2 to +2 days surrounding the earthquakes may be present).

While Fig. $3 \mathrm{c}$ is straightforward to interpret and shows important absolute trends, it is nevertheless somewhat deficient in that it obscures how Pc1 occurrence behaves compared with its typical behavior. To address this issue, we derive the relative probability of $\mathrm{Pc} 1$ occurrence similar to Bortnik et al. (2008). First, the entire set of identified Pc1 pulsations is binned into 12 LT bins. The resulting histogram is shown in Fig. 3a, and is binned similarly in LT to the superposed epoch plot of Fig. 3c, and again underscores the strong diurnal variation. The histogram in Fig. $3 \mathrm{a}$ is divided by the total number of days of instrument operation (corrected for any outages), to effectively obtain a long-term, daily occurrence probability of Pc1 pulsations. Similarly, the superposed epoch plot in Fig. $3 \mathrm{c}$ is normalized by the total number of earthquake events (434) to obtain a time-dependent probability of occurrence, and then each column is normalized by the daily occurrence probability, resulting essentially in a distribution of Pc1 pulsation occurrence probability, as a function of $\Delta T$ and LT, relative to the long-term occurrence probability.

The resulting plot is shown in Fig. 3d, where it has now become evident that the relative probability of occurrence of Pc1 pulsations is significantly higher during the day time hours, $\sim 5-15$ days prior to the earthquake occurrence, by a factor of $\sim 2-6$. A few points should be noted about this figure: firstly, the majority of values in the figure (especially near the night and dawn) are $\sim 1$, indicating that the occurrence probability at those $\mathrm{LT}$ and $\Delta T$ bins is roughly similar to the long-term occurrence probability, and hence is likely unaffected by the earthquake events. Secondly, there is a clear difference in relative probability values prior to (negative $\Delta T$ ), and post-earthquake occurrence (positive $\Delta T$ ). In the former region $(\Delta T<0)$, there is a clear increase in relative occurrence probability, primarily in the daytime hours, whereas in the latter region $(\Delta T>0)$ the relative occurrence probability visibly drops to its long-term average. The relative occurrence at $\Delta T<0$ is not only large, but seems to be increasing as the earthquake approaches, reaching a maximum at $\Delta T \sim-5$ days, and then decreasing again. Furthermore, it is not only a matter of a few isolated $\Delta T$-LT bins that have increased values, but a well-defined, connected region of bins spanning the entire day side, over a number of consecutive days.

For clarity, we have integrated the number of Pc1 pulsation in Fig. 3c from 08:00 to 16:00, and show the resulting line plot in Fig. 3e. Similarly, we averaged the relative occurrence probability in Fig. 3d over the same LT interval and show the line plot in Fig. 3f. In both panels (e) and (f), the increase of $\mathrm{Pc} 1$ pulsations during midday, 5-15 days ahead of the earthquake is clearly evident. We note that the 5-day timescale is consistent with the statistical ionospheric anomalies reported by Liu et al. (2006).

If we assume that this is a real, physical effect, it could be explained by the fact that during daytime hours the natural occurrence of $\mathrm{Pc} 1$ pulsations is relatively low, and hence if there is an addition of even a small number of $\mathrm{Pc} 1$ pulsations at all local times, it would show up as a much larger percentage change during the daytime, than during the night. On the other hand, since we are dealing with a smaller number of Pc1 events during the day (although not as small as Fig. 3a might indicate, since we superpose 434 individual events), the increased probability observed at $\Delta T=-5$ to -15 might be simply coincidental, and not physically significant. In the following section, we attempt to quantify the significance of the relative probability increase evident in Fig. 3d.

\section{Tests of significance}

The procedure for quantifying significance is illustrated in Fig. 4. In order to compare the probability of obtaining the results of Fig. 3d (reproduced in Fig. 4a) with pure chance, we generate a sequence of 434 events, distributed randomly during the Pc1 observation period (February 1999July 2006), and perform the same analysis described in Sect. 4 above. The results are displayed in Fig. 4b, and show that the natural variation of relative probability is certainly higher during daytime hours, but is significantly smaller $(<2)$ and more sporadic than the plot of Fig. 4a. As expected, there is no correlation of $\mathrm{Pc} 1$ occurrence probability with the random event sequence.

Next, we repeat the superposed epoch analysis with a random event sequence (as described above) 300 times, so that we are left with 300 plots like those of Fig. 4b. Since there is no correlation with the random event sequence, the values from every row of the random plots can be grouped together, giving us a sequence of $(300 \times 40=) 12000$ points for each of the 12 LT bins. It is these sequences which will be used to assess the significance of the results in Fig. $4 \mathrm{a}$.

Each of the 12 row sequences is tested for consistency with one of several specified distributions using the AndersonDarling test (Anderson and Darling, 1952). This test is particularly useful for our purposes, since it gives more weight to the tails than the comparable Kolmogorov-Smirnov test, and has been shown to be one of the best statistics for detecting departures from normality (Stephens, 1974). Using this test, each of the 12 row sequences turns out to be extremely consistent with a normal distribution, at the $5 \%, 1 \%, 0.1 \%$ (and lower) level. As such, it is meaningful to derive the mean $\mu$ and standard deviation $\sigma$ of each sequence, which are shown as a function of local time in Fig. 4d. Not surprisingly, the standard deviation in the 12:00-14:00 LT bin 
EQ

(a) Earthquakes $=434$

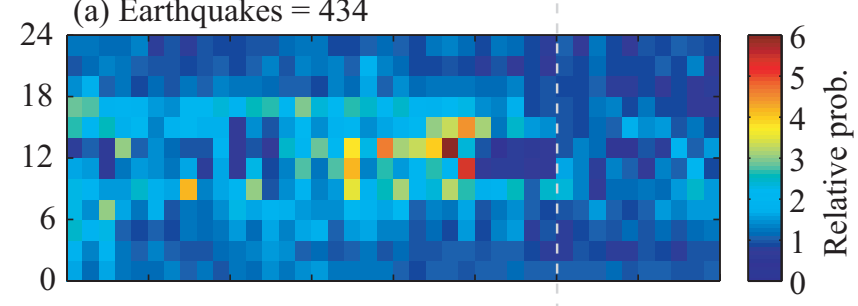

(b) Random events $=434$

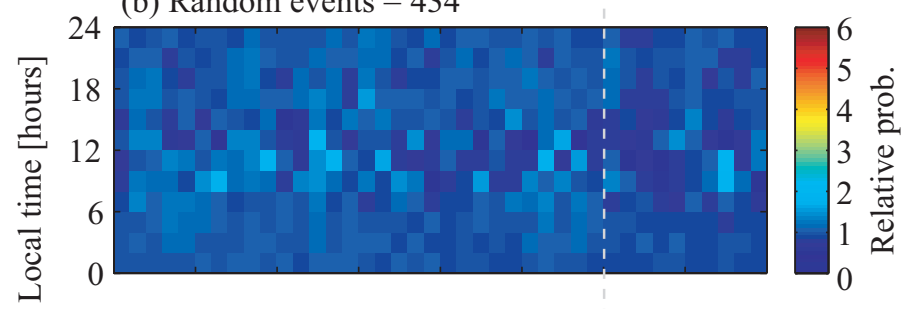

(c) "Normalized" Earthquakes $=434$

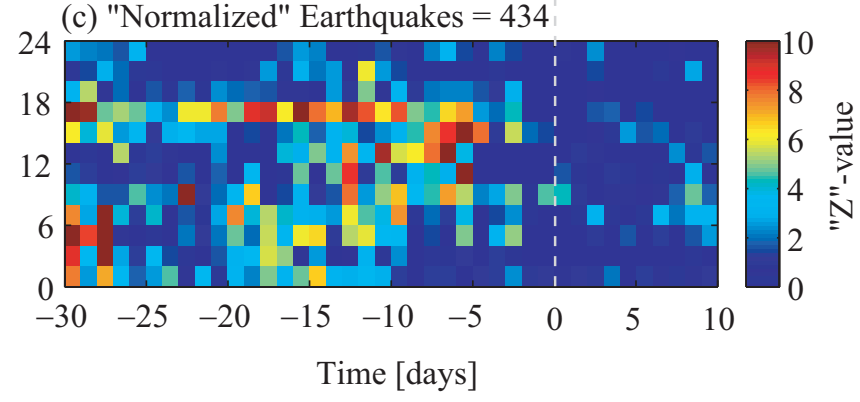

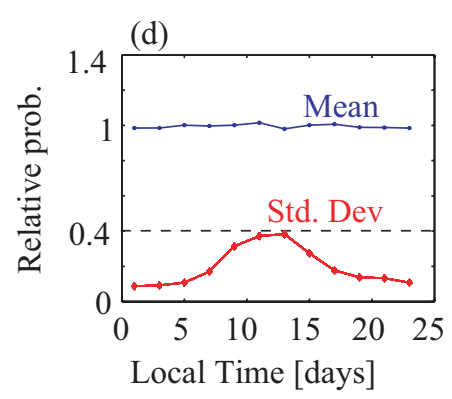

(e)

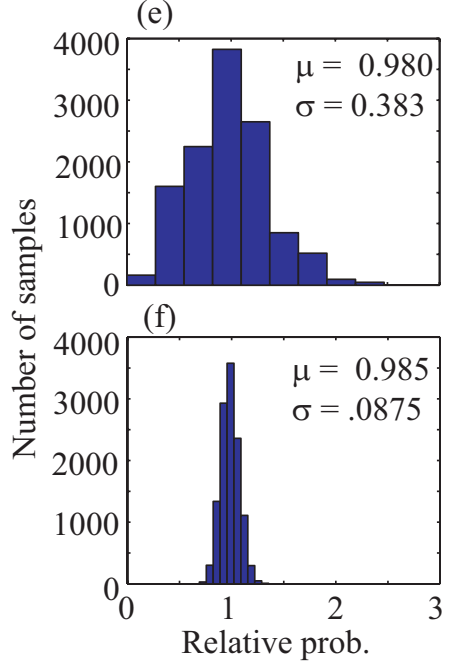

Fig. 4. Significance test of Pc1 observations. (a) Relative probability of observing Pc1 pulsations with respect to earthquakes, (similar to Fig. 3d), (b) same as (a) with respect to 434 random events, (c) normalized relative probability (d) mean and standard deviation of random statistical sample, (e) histogram plot of random event distribution for LT=12:00-14:00 (highest variance), and (f) same as (e) for $\mathrm{LT}=$ 00:00-02:00 (lowest variance).

turns out to be largest $(\sigma=0.383$ ), coincident with the minimum of Pc1 event occurrence in Fig. 3a. On the other hand, the mean is $\sim 1$ at all local times. We note that the choice of producing 300 random-event sequences was made so that the standard error of the mean would be $\sim 0.01 \sigma$ (i.e. $\sigma / \sqrt{N}$ where $N=12000$ ). For reference, a 10-bar histogram of the $\mathrm{Pc} 1$ pulsation relative probability is plotted in Figs. $4 \mathrm{e}$ and $\mathrm{f}$, corresponding to the highest and lowest $\sigma$ LT bins, respectively.

Each of the columns of Fig. 4a is normalized by subtracting the appropriate LT mean, and dividing by the corresponding standard deviation (Fig. 4b), to give a plot of " $Z$ " values as a function of $\mathrm{LT}$ and $\Delta T$, i.e. relative probability of Pc1 occurrence, measured in standard deviations away from the mean. The results, shown in Fig. 4c, indicate that the daytime increase in Pc1 relative probability, at $\Delta T \sim 5$ to 15 prior to the earthquake is very significant $(Z>4 \sigma)$ and is very unlikely due to chance. In addition to the daytime feature which was clear in Fig. 4a, there are additional regions of high $Z$ that appear near the dusk and night sides, including a region at $\Delta T<-27$ which may or may not be associated with the earthquake. Again, there is a clear difference in the $\mathrm{Z}$ values between regions preceding the earthquake $(\Delta T<0)$ and those that occur after the earthquake $(\Delta T>0)$.

\section{Effects of declustering}

It has long been established that earthquakes tend to occur in well-defined clusters (Omori, 1895), which would affect the type of analysis presented above (since a single Pc1 pulsation could be registered in association with multiple, closelyspaced earthquakes). This effect could make the correlation appear far more significant than it actually is in reality, and further result in time-smearing.

In order to assess the impact of earthquake clustering on our analysis, we used the simple, 2-parameter declustering algorithm developed by Reasenberg (1985), to remove aftershocks in our earthquake catalog. The results are shown in Fig. 5, where panel (a) is again a reproduction of the relative 
(a)

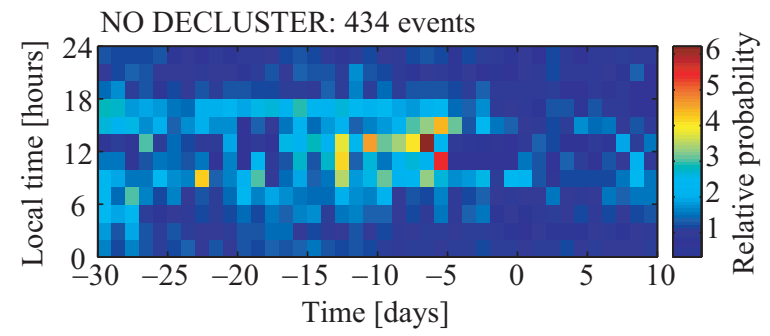

(c)

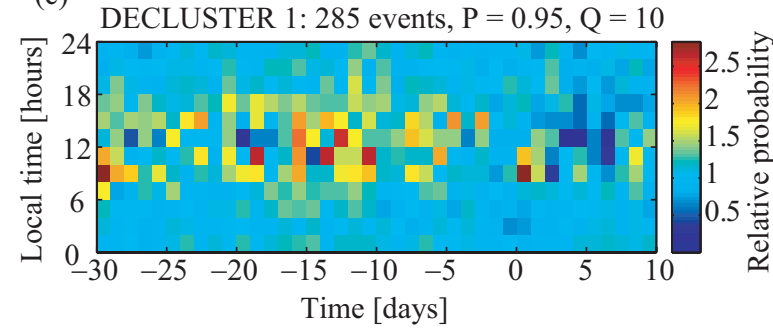

(e)

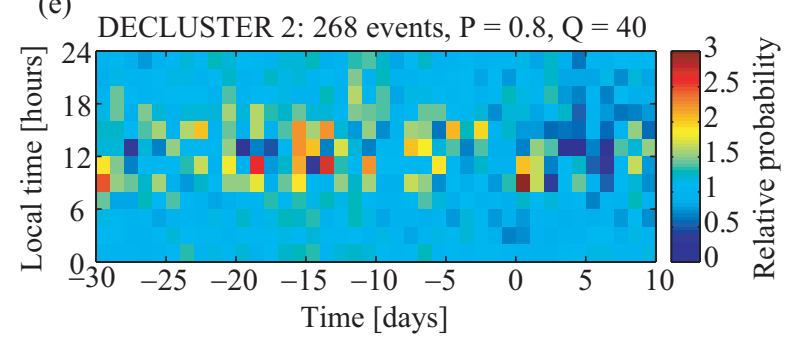

(b)

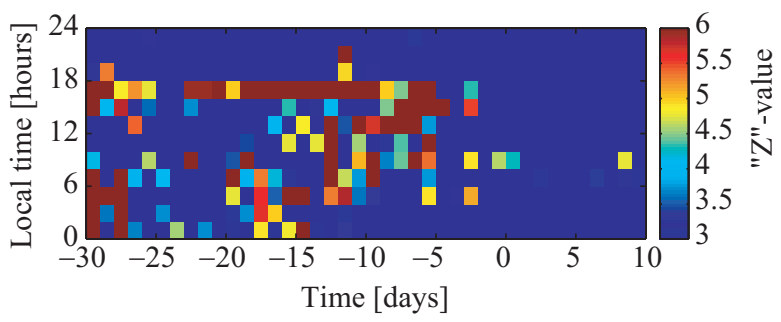

(d)

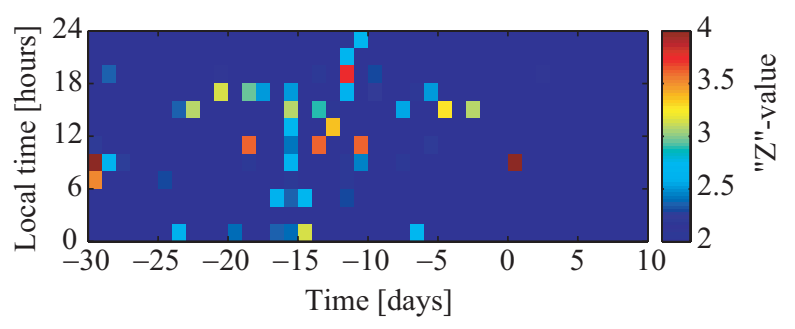

(f)

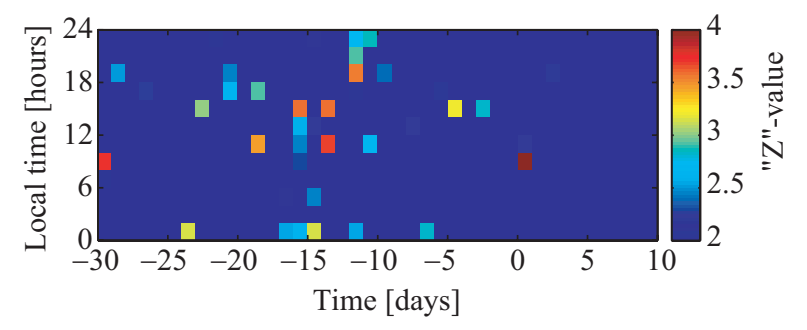

Fig. 5. Effect of declustering on significance. (a) No declustering (same as Fig. 4a), (b) significance of (a) (same as Fig. 4d), (c) typical declustering level, and (d) significance of (c), (e) aggressive declustering and (f) significance of (e).

probability plot of Figs. 3d and 4a, and panel (b) is the corresponding Z-value plot, similar to Fig. 4c but clipped between $Z=3-6$ to show only significant values.

In panel (c), we use the declustering algorithm with typical values of $P=0.95$ and $Q=10$, where $P$ and $Q$ determine the sizes of the spatial and temporal windows, respectively. The resulting earthquake list contains only 285 events, and the resulting distribution again shows a daytime maximum prior to the earthquake event. Comparing with panel (a), it is evident that the relative probability has decreased substantially, showing that much of the correlation was due to the aftershocks. The region of high relative probability has shifted to slightly earlier times, now peaking in the region $\Delta T \sim 10$ to 20 days prior to the main shock. Also evident is the appearance of a slight drop in relative probability after the earthquake, at $\Delta T \sim 5$ days. To assess the significance of the results in panel (c), we again compute the sequence of relative probability values for each LT bin, this time using 285 random events for statistical uniformity, and repeating the procedure 300 times as before. The Anderson-Darling test is again applied to ensure normality. The normalized plot of
Z-values for the typical declustering parameters is shown in panel (d), where it is clear that there are regions of high $Z$, albeit much lower than those in panel (b). We have clipped the color scale above $Z=2$, since $2 \sigma$ roughly corresponds to the $5 \%$ significance level, so everything above it can be considered significant. Interestingly, in panel (d) the region of high $Z$ is not confined only to the day side, but extends across the entire LT range in the region of $\Delta T \sim-20$ to -10 days. We again note that there is a clear contrast in the $\mathrm{Z}$ values between regions preceding the earthquake $(\Delta T<0)$ and those that occur after the earthquake $(\Delta T>0)$.

In the bottom row of Fig. 5, we perform a more aggressive declustering than normal, with $P=0.8$ and $Q=40$. The resulting list of earthquakes contains 268 events, and the resulting relative probability plot and Z-value plot shown in panels (e) and (f) appear very similar to those of panels (c) and (d), respectively, with only minor differences. The region of increased Pc1 probability remains at roughly $\Delta T \sim-15$ to -10 days, and the contrast in $\mathrm{Z}$-value behavior between $\Delta T<0$ and $\Delta T>0$ is apparent. 


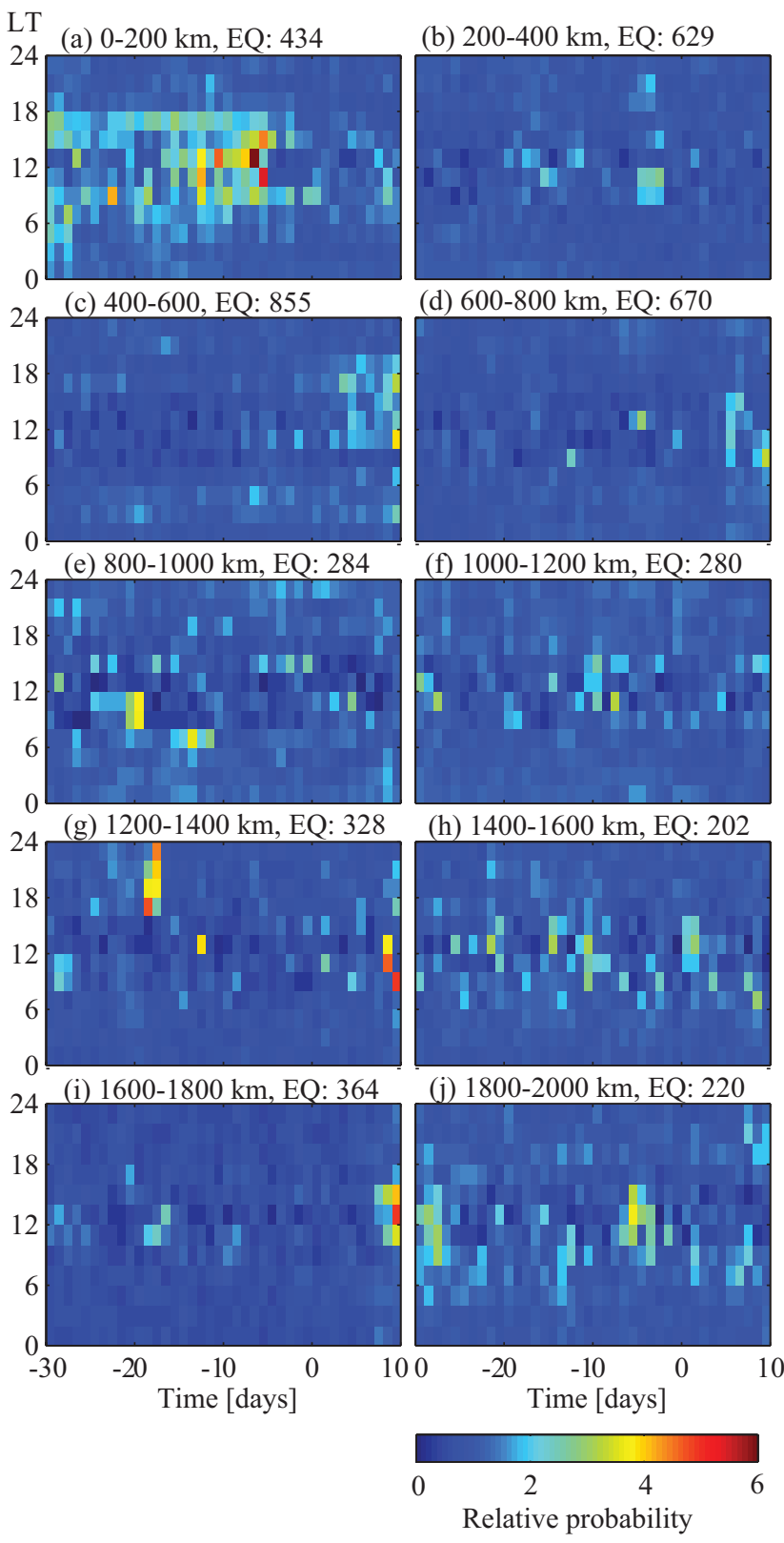

Fig. 6. The effect of distance upon the relative occurrence probability of Pc1 pulsations in relation with earthquakes. Radial distance ranges increase in increments of $200 \mathrm{~km}$ from 0-200 km (a), to $1800-2000 \mathrm{~km}(\mathbf{j})$. The number of earthquakes is indicated in the title of each panel.

\section{$7 \quad$ Effects of distance}

To establish the spatial locality of our correlation, we examine the effect of distance upon the relative occurrence probability of Pc1 pulsations, relative to earthquakes in Fig. 6. In panel (a) we show the relative occurrence probability similar to Figs. 3d, 4a, and 5a, where earthquakes have only been included in the radial range of $0-200 \mathrm{~km}$ around the PKD magnetometer. In subsequent panels, the radial range within which earthquakes are selected for the superposed epoch analysis is increased in increments of $200 \mathrm{~km}$, from $200-400 \mathrm{~km}$ in panel (b) to $1800-2000 \mathrm{~km}$ in panel (j). Although there are spurious regions of enhanced relative probability in many of the figures, such as panel (g) $\Delta t \sim-18$ days and $\Delta t \sim+10$ days, or panel (i) $\Delta t \sim+10$ days, the relative probability magnitudes are generally smaller than those in panel (a), and do not seem to show a systematic grouping as do those in panel (a). It thus appears as though the enhancement of the $\mathrm{Pc} 1$ pulsations relative to the earthquake occurrence is limited to $\sim 200 \mathrm{~km}$ around the magnetometer, or possibly slightly further as in panel (b). We do also note that the number of earthquakes within each radial range does vary, making the statistics within each panel non-uniform (although the variation is confined to a factor of $\sim 2$ ), so again we urge the reader to treat these results as being preliminary.

\section{Earthquake probability}

As a final analysis, we invert the superposed epoch procedure presented in Sect. 4, selecting only those Pc1 pulsations that occur between the local-times of 08:00 of 16:00 (giving 423 Pc1's) as the fiducial times, and examining the probability of earthquake occurrence within a window of -10 days to +30 days about each midday Pc1 pulsation.

Earthquakes are binned as a function of time relative to the Pc1 pulsation $\left(\triangle T_{E Q}\right)$, in 2-day bins, and magnitude, in $0.5 \mathrm{M}$ bins, from $M=3.0$ to $M=5.5$. After the initial distribution is obtained (analogous to Fig. 3c), it is normalized by the number of Pc1 pulsations, and number of days per bin (2 days/bin), resulting in a time-dependent occurrence probability. The number of earthquakes in our observing period (February 1999-July 2006) is binned according to magnitude (analogous to Fig. 3a) and divided by the number of observing days, to obtain a long-term daily occurrence probability. The earthquake occurrence probability distribution is normalized column-wise by the daily occurrence probability, resulting in a relative probability of occurrence plot (analogous to Fig. 3d) which is shown in Fig. 7a. This figure indicates that, on average, the occurrence probability of a M4.0 M5.5 earthquake is roughly 5 times higher following a midday Pc1 pulsation, than it is normally (though the number of large earthquakes is rather low, cf. Fig. 3b). The occurrence probabilities of smaller earthquakes are also larger by factors of $\sim 2-4$ in the week following a midday Pc1 pulsation.

For purposes of comparison, we perform the same analysis described above, with a similar sequence of events, but this time randomly generated (analogous to Fig. 5b). The results are shown in Fig. 7b, and exhibit spurious regions of enhanced earthquake relative occurrence probability, that do not appear to be systematic as in panel (a). Furthermore, the 
values are on the order of $\sim 2$, significantly lower than the values in panel (a).

Since it is well-known that Pc1 pulsations are associated with enhanced geomagnetic activity (Wentworth, 1964; Heacock and Kivinen, 1972; Bortnik et al., 2008), we test the possible connection between geomagnetic activity and earthquakes directly. Shown in panel (c) is the result of a superposed epoch analysis, performed as above, but using a sequence of 220 storm sudden commencements (SSC's) as the fiducial time. The results do not show any noticeable enhancement in the relative occurrence probability of earthquakes associated with SSC's. We have also used an automatic algorithm to pick out 24 isolated storms directly from the $D_{s t}$ index (Bortnik et al., 2008) and use the storm times as the fiducial times, performing the same superposed epoch analysis. The results (not shown), do not seem to show any correlation between geomagnetic storms and earthquakes. We probed the connection between $D_{s t}$ depressions and global seismicity further with a number of analyses (not shown), and again found no significant correlation.

The results presented in Fig. 7 suggest that even though $\mathrm{Pc} 1$ pulsations are strongly correlated with geomagnetic activity, and are also apparently well-correlated with earthquakes, geomagnetic activity and earthquakes do not show any direct correlation. If the correlation of Pc1 pulsations and earthquakes is indeed real, as it appears to be, then those midday Pc1 pulsations that precede earthquakes probably originate from a source other than geomagnetic storms. The options which seem most likely are that the Pc1 pulsations are either generated within the earth, due to electric currents, or are generated by triggers other than geomagnetic storms, such as small storms, substorms, pressure pulses in the solar wind, or drifting fluxes of anisotropic protons from the plasmasheet (Olson and Lee, 1983; Kangas et al., 1986; Engebretson et al., 2002) and it is indeed the modification of the bottomside ionosphere prior to the earthquake that alters the transmission of $\mathrm{Pc} 1$ pulsations from the ionosphere to the ground. As noted above, the timescales of Pc1 probability increase correspond to those of ionospheric modification (Liu et al., 2006), hinting at a possible connection.

To assess whether the presence of midday Pc1 pulsations might be useful as a reliable indicator of imminent earthquakes, we attempted to quantify the number of false positive and negative events. In order to do this, we again isolated only those Pc1 pulsations observed between 08:00 and 16:00, and counted the number of $\mathrm{Pc} 1$ pulsations that were followed by an earthquake within 2 weeks (within $200 \mathrm{~km}, \mathrm{M} \geq 3$ ) and vice versa. The results of this analysis are shown in Table 1 , and indicate that roughly two thirds of $\mathrm{Pc} 1$ pulsations were followed by at least one earthquake event, a roughly similar fraction of earthquake events ( 274 of 434 ) were preceded by at least one Pc1 pulsation. For comparison, we performed a similar analysis on a random event sample of the same size as the earthquake list, repeated 300 times, and obtain an estimate for the mean and standard deviation (also shown in Ta-
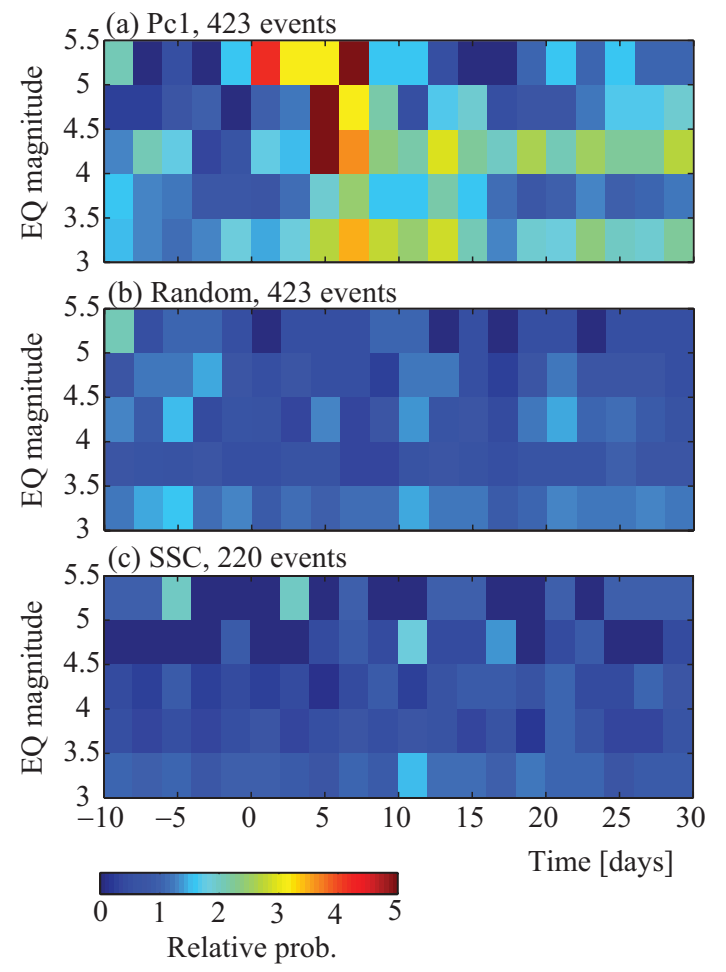

Fig. 7. Superposed epoch analysis of relative probability of observing earthquakes, parameterized by time and earthquake magnitude, relative to (a) mid-day Pc1 events, (b) random event sequence, and (c) Storm sudden commencements.

ble 1). While the random events occur roughly independently of Pc1 pulsations, it is interesting to note that it is quite likely (84\%) to observe a random event, given a randomly selected midday Pc1 pulsation. This is most likely a consequence of the tendency of $\mathrm{Pc} 1$ pulsations to occur in clusters.

If the aggressively declustered earthquake catalog (e.g. Sect. 6, Fig. 5e, f) is used, results show that the number of earthquakes that is preceded by Pc1 events becomes comparable to random events, and similarly for earthquakes that follow Pc1 pulsations. It appears that merely observing midday Pc1 pulsations does not significantly increase the likelihood that an earthquake will follow, but it is the total number of midday Pc1 pulsations which increase in advance of earthquakes, as shown above.

\section{Conclusions}

In this paper we examined the association between earthquakes and Pc1 pulsations observed at a low-latitude station in Parkfield, California. The period under examination was $\sim 7.5$ years in total, from February 1999 to July 2006, and we used an automatic identification algorithm to extract information on Pc1 pulsations from the magnetometer data. These 
Table 1. Percentage of midday Pc1 pulsations with, and without, an earthquake ensuing within 2 weeks, and vice versa. Columns correspond to the full earthquake catalog $(M \geq 3$, within $200 \mathrm{~km})$, the mean $\mu$ and standard deviation $\sigma$ of 300 similar random event samples.

\begin{tabular}{lcccc}
\hline & Full & Random 434 $(\mu, \sigma)$ & Declustered & Random 268 $(\mu, \sigma)$ \\
\hline EQ's with Pc1 & $63.1 \%(274 / 334)$ & $52.5 \%, 2.5 \%$ & $50.0 \%$ & $52.5 \%, 3.0 \%$ \\
EQ's without Pc1 & $36.9 \%(160 / 334)$ & $47.5 \%, 2.5 \%$ & $50.0 \%$ & $47.5 \%, 3.0 \%$ \\
Pc1 with EQ's & $72.6 \%(307 / 423)$ & $87.7 \%, 3.6 \%$ & $72.6 \%$ & $75.6 \%, 5.1 \%$ \\
Pc1 without EQ's & $27.4 \%(116 / 423)$ & $9.8 \%, 3.6 \%$ & $27.4 \%$ & $24.4 \%, 5.1 \%$ \\
\hline
\end{tabular}

pulsations were then statistically correlated to earthquakes from the USGS NEIC catalog within a radius of $<200 \mathrm{~km}$ around the magnetometer, and $\mathrm{M}>3.0$.

It was shown that the superposed epoch analysis by itself did not reveal any visible correlation between the Pc1 pulsations and earthquakes. However, when the distribution of Pc1's was normalized by the daily occurrence probability, an occurrence peak emerged on the day side, $\sim 5-15$ days prior to the earthquake. The significance of this occurrence peak was tested by comparing it against random sequences of events. Results show that the Pc1 distribution, and precursory daytime maximum, are very significant, and are most probably not due to chance.

We then tested the effect of declustering our earthquake catalog and repeating the significance tests above. Two declustering analyses were analyzed, one for a typical removal of aftershocks, and one for an aggressive removal. Results indicate that even though the significance levels did decrease substantially, there were nevertheless statistically significant regions of enhanced Pc1 occurrence several days ahead of the earthquakes. It is noteworthy that the Pc1 occurrence prior to, and following earthquakes, always showed a dramatic difference.

Finally, we plotted the relative probability of earthquakes in response to daytime $\mathrm{Pc} 1$ pulsations, and compared against similar relative probability plots made against a random event sequence, a list of storm sudden commencements, and a list of isolated geomagnetic storms. It was shown that the earthquake probability only increased in response to day time Pc1 pulsations, by factors of $\sim 3-5$ depending on the magnitude of the earthquake. It was also interesting to note that there did not appear to be any significant correlation between geomagnetic activity and earthquake occurrence, begging the question: what was the source of the Pc1 pulsations, if not geomagnetic activity? One model is that preseismic activity might have led to ionospheric modifications which affected the propagation characteristics of (quiet-time) Pc1 pulsations, although other explanations are by no means excluded by the present analysis alone.

Although the results presented herein are certainly suggestive, we nevertheless remain cautious in generalizing the conclusions, until these results can be repeated with data from other periods and especially other (low-latitude) locations around the world. If these results are indeed found to be re- peatable, a reliable discriminator would need to be developed to distinguish those Pc1 pulsations that precede earthquakes from those that do not, if such a method is to be useful in earthquake early warning systems.

Acknowledgements. This work was supported by QuakeFinder LLC., Stellar Solutions Inc., the California Space Authority (CSA), and NASA grant NNG04GD16A. Magnetometer data was obtained from the Northern California Earthquake Data Center (NCEDC), contributed by the Berkeley Seismological Laboratory, University of California, Berkeley. The authors would like to thank Celeste V. Ford for her ongoing help and support.

Topical Editor M. Pinnock thanks two anonymous referees for their help in evaluating this paper.

\section{References}

Althouse, E. L. and Davis, J. R.: Five station observations of Pc1 micropulsation propagation, J. Geophys. Res., 83, 132-144, 1978.

Anderson, T. W. and Darling, D. A.: Asymptotic theory of certain "goodness-of-fit" criteria based on stochastic processes, Ann. Math. Stat., 23, 193-212, 1952.

Bernardi, A., Fraser-Smith, A. C., McGill, P. R., and Villard Jr., O. G.: ULF magnetic field measurements near the epicenter of the Ms 7.1 Loma Prieta earthquake, Phys. Earth Planet. Inter., 68, 45-63, 1991.

Bortnik, J., Cutler, J. W., Dunson, C., and Bleier, T. E.: An automatic wave detection algorithm applied to Pc1 pulsations, J. Geophys. Res., 112, A04204, doi:10.1029/2006JA011900, 2007.

Bortnik, J., Cutler, J. W., Dunson, C., Bleier, T. E., and McPherron, R. L.: Characteristics of low latitude Pc1 pulsations during geomagnetic storms, J. Geophys. Res., 113, A04201, doi:10.1029/2007JA012867, 2008.

Brace, W. F., Orange, A. S., and Madden, T. R.: The effect of pressure on the electrical resistivity of water-saturated crystalline rock, J. Geophys. Res., 70, 5669-5678, 1965.

Bräysy, T., Mursula, K., and Marklund, G.: Ion cyclotron waves during a great magnetic storm observed by Freja double-probe electric field instrument, J. Geophys. Res., 103(A3), 4145-4155, 1998.

Campbell, W. H. and Stiltner, E. C.: Some characteristics of geomagnetic pulsations at frequencies near $1 \mathrm{c} / \mathrm{s}$, Radio Sci. J. Res. NBS/USNC-URSI, 69D(8), 1117-1132, 1965.

Clilverd, M. A., Rodger, C. J., and Thomson, N. R.: Investigating seismoionospheric effects on a long subionospheric path, J. Geophys. Res., 104(A12), 28 171-28 179, 1999. 
Cornwall, J. M.: Cyclotron instabilities and electromagnetic emission in the Ultra Low Frequency and Very Low Frequency ranges, J. Geophys. Res., 70(1), 61-69, 1965.

Derr, J. S.: Earthquake lights - review of observations and present theories, B. Seis. Soc. Am., 63(6), 2177-2187, 1973.

Dogloglou-Revelioti, E. and Varotsos, P.: Thermally stimulated currents in rocks, J. Geophys., 59, 177-182, 1986.

Engebretson, M. J., Peterson, W. K., Posch, J. L., Klatt, M. R., Anderson, B. J., Russell, C. T., Singer, H. J., Arnoldy, R. L., and Fukunishi, H.: Observations of two types of Pc 1-2 pulsations in the outer dayside magnetosphere, J. Geophys. Res., 107(A12), 1451, doi:10.1029/2001JA000198, 2002.

Fraser, B. J.: Temporal variations in Pc1 geomagnetic micropulsations, Plant. Space Sci., 16, 111-124, 1968.

Fraser, B. J. and Nguyen, T. S.: Is the plasmapause a preferred source region of electromagnetic ion cyclotron waves in the magnetosphere?, J. Atmos. Sol. Terr. Phys., 63, 1225-1247, 2001.

Fraser-Smith, A. C.: Some statistics on Pc1 geomagnetic micropulsation occurrence at middle latitudes: inverse relation with sunspot cycle and semiannual period, J. Geophys. Res., 75, 4735-4745, 1970.

Fraser-Smith, A. C., Bernardi, A., McGill, P. R., Ladd, M. E., Helliwell, R. A., and Villard Jr., O. G.: Low-frequency magnetic field measurements near the epicenter of the Ms 7.1 Loma Prieta earthquake, Geophys. Res. Lett., 17, 1465-1468, 1990.

Field, E. C. and Greifinger, C.: Transmission of geomagnetic micropulsations through the ionosphere and lower exosphere, J. Geophys. Res., 70, 4885-4899, 1965.

Field, E. C. and Greifinger, C.: Equatorial transmission of geomagnetic micropulsations through the ionosphere and lower exosphere, J. Geophys. Res., 71, 3223-3232, 1966.

Freund, F. T.: Pre-earthquake signals - Part I: Deviatoric stresses turn rocks into a source of electric currents, Nat. Hazards Earth Syst. Sci., 7, 535-541, 2007,

http://www.nat-hazards-earth-syst-sci.net/7/535/2007/.

Hanks, T. C.: Constraints on the dilatancy-diffusion model of the earthquake mechanism, J. Geophys. Res., 79, 3023-3026, 1974.

Hayakawa, M. and Molchanov, O. A.: Achievements of NASDA's earthquake remote sensing frontier project, TAO, 15(3), 311327, 2004.

Hayakwa, M., Pulinets, S., Parrot, M., and Molchanov, O. A.: Recent progress in seismo electromagnetics and related phenomena, Physics and Chemistry of the Earth, Parts A/B/C, 31(4-9) (special issue), 129-496, 2006.

Hayakawa, M., Hattori, K., and Ohta, K.: Monitoring of ULF (ultralow-frequency) geomagnetic variations associated with earthquakes, Sensors, 7, 1108-1122, 2007.

Heacock, R. R. and Kivinen: Relation of Pc1 micropulsations to the ring current and geomagnetic storms, J. Geophys. Res., 77(34), 6746-6760, 1972.

Hendersen, T. R., Sonwalkar, V. S., Helliwell, R. A., Inan, U. S., and Fraser-Smith, A. C.: A search for ELF/VLF emissions induced by earthquakes as observed in the ionosphere by the DE 2 satellite, J. Geophys. Res., 98, 9503-9514, 1993.

Hsu, T.-S. and McPherron, R. L.: An evaluation of the statistical significance of the association between northward turnings of the interplanetary magnetic field and substorm expansion onsets, J. Goephys. Res., 107(A11), 1398, doi:10.1029/2000JA000125, 2002.
Ishido, T. and Mizutani, M.: Experimental and theoretical basis of electrokinetic phenomena to rock-water systems and its applicaations to geophysics, J. Geophys. Res., 86, 1763-1775, 1981.

Jacobs, J. A.: Geomagnetic micropulsations, Springer-Verlag, 1970.

Johnson, J. R. and Cheng, C. Z.: Can ion cyclotron waves propagate to the ground?, Geophys. Res. Lett., 26(6), 671-674, 1999.

Johnston, M. J. S.: Review of magnetic and electric field effects near active faults and volcanos in the USA, Phys. Earth Planet. Inter., 57, 47-63, 1989.

Kalashnikov, A. D.: Potentialities of magnetometric methods for the problem of earthquake forerunners. Tr. Geofiz. Inst., Akad. Naul. SSSR., 25, 180-182, 1954.

Kangas, J., Guglielmi, A., and Pokhotelov, O.: Morphology and physics of short-period magnetic pulsations, Space Sci. Rev., 83, 435-512, 1998.

Larkina, V. I., Nalivayko, A. V., Gershenzon, N. I., Gokhberg, M. B., Liperovskiy, V. A., and Shalimov, S. L.: Observations of VLF emissions related with seismic activity, on the Interkosmos-19 satellite, Geomagn. Aeron., 23, 684-687, 1983.

Liu, J. Y., Chen, Y. I., Chuo, Y. J., and Chen, C. S.: A statistical investigation of preearthquake ionospheric anomaly, J. Geophys. Res., 111, A05304, doi:10.1029/2005JA011333, 2006.

Manchester, R. N.: Propagation of Pc1 micropulsations from high to low latitudes, J. Geophys. Res., 71, 3749-3754, 1966.

Manchester, R. N.: Correction of Pc1 micropulsations at spaced stations, J. Geophys. Res., 73(11), 3549-3556, 1968.

Molchanov, O., Kulchitsky, A., and Hayakawa, M.: Inductive seismo-electromagnetic effect in relation to seismogenic ULF emission, Nat. Hazards Earth Syst. Sci., 1, 61-67, 2001, http://www.nat-hazards-earth-syst-sci.net/1/61/2001/.

Morgan, F. D., Williams, E. R., and Madden, T. R.: Streaming potential properties of Westerly granite with applications, J. Geophys. Res., 94, 12 449-12 461, 1976.

Nagata, T.: The effect of a uniaxial compression on remnant magnetizations of igneous rocks, Pure Appl. Geophys., 78, 100-109, 1970a.

Nagata, T.: Basic magnetic properties of rocks under the effect of mechanical stresses, Tectonophysics, 9, 167-195, 1970b.

Olson, J. V. and Lee, L. C.: Pc1 wave generation by sudden impulses, Planet. Space Sci., 31, 295-302, 1983.

Omori, F.: On the aftershocks of earthquakes, J. College Sci. Imper. Univ. Tokyo, 7, 111, 1895.

Park, S. K., Johnston, M. J. S., Madden, T. R., Morgan, D. F., and Morrison, H. F.: Electromagnetic precursors to earthquakes in the ULF band: A review of observations and mechanisms, Rev. Geophys., 31(2), 117-132, 1993.

Parrot, M. and Johnston, M. J. S. (Eds.): Seismoelectromagnetic effects, Phys. Earth Planet. Inter., 57, 1-177, 1989.

Parrot, M.: Special issue of plantary and space science 'DEMETER', Planet. Space Sci., 54, 411 , doi:10.1016/j.pss.2005.10.012, 2006.

Parrot, M. and Lefeuvre, F.: Correlation between GEOS VLF emissions and earthquakes, Ann. Geophys., 3(6), 737-748, 1985.

Parrot, M., Achache, J., Berthelier, J. J., Blanc, E., Deschamps, A., Lefeuvre, F., Menvielle, M., Plantet, J. L., Tarits, P., and Villain, J. P.: High-frequency seismo-electromagnetic effects, Phys. Earth Plant. Inter., 77, 65-83, 1993.

Pulinets, S. A., Legen'ka, A. D., Gaivoronskaya, T. V., and Depuev, 
V. Kh.: Main phenomenological features of ionospheric precursors of strong earthquakes, J. Atmos. Sol. Terr. Phys., 65, 13371347, 2003.

Pulinets, S. A. and Boyarchuk, K. A.: Ionospheric precursors of earthquakes, Springer, Heidelberg, New York, 2004.

Reasenberg, P.: Second-order moment of central California seismicity, 1969-1982, J. Geophys. Res., 90(B7), 5479-5495, 1985.

Rodger, C. J., Thomson, N. R., and Dowden, R. L.: A search for ELF/VLF activity associated with earthquakes using ISI satellite data, J. Geophys. Res., 101(A6), 13 369-13 378, 1996.

Rodger, C. J., Clilverd, M. A., and Thomson, N. R.: Modeling subionospheric VLF signal perturbations associated with earthquakes, Radio Sci., 34(5), 1177-1185, 1999.
Stephens, M. A.: EDF statistics for goodness of fit and some comparisons, J. Am. Stat. Assoc., 69, 730-737, 1974.

Webster, D. J. and Fraser, B. J.: Source structure and dynamics of Pc 1 pulsations at low latitudes, Planet. Space Sci., 32(8), 935947, 1984.

Wentworth, R. C.: Enhancement of hydromagnetic emissions after geomagnetic storms, J. Geophys. Res., 69(11), 2291-2298, 1964.

Zhao, Y. L. and Qian, F. Y.: Geoelectric precursors to strong earthquakes in China, Tectonophysics, 233, 99-113, 1994. 